\title{
Clinical Fluid Therapy in the Perioperative Setting
}

\author{
Robert G. Hahn (editor). Cambridge University Press, 2017. ISBN-13: 9781107119550
}

\author{
Victor Tran, MD
}

Received: 11 May 2017/Accepted: 30 May 2017/Published online: 14 June 2017

(C) Canadian Anesthesiologists' Society 2017

Intravenous fluid is one of the first therapies physicians learn to administer during their medical training. Indeed, the approach to fluid therapy has evolved from viewing it as a straightforward trial-and-error intervention to an emerging evidence-based practice. The options for fluid therapy have expanded from simple isotonic sodium chloride solutions to complex artificial (i.e., hydroxyethyl starches) or human-derived (i.e., albumin) formulations. This evolution has led to a question that many practitioners ask themselves on a regular basis - i.e., which fluid should I use for my patient and why?

This second-edition, 277-page book entitled, Clinical Fluid Therapy in the Perioperative Setting, by Robert Hahn attempts to consolidate current knowledge on fluid therapy to provide guidance as to its optimal use. The multiauthored book includes valuable and significant contributions from European, North American, African, and Australian authors. Despite this large variety of contributing authors, the book maintains a smooth, easyto-read flow as it takes the reader through its four major sections. The sections are based on the various fluid types, basic science and physiology, techniques in administration and monitoring, and applications in the perioperative clinical setting.

The book's first section opens with a brief chapter on basic fluid types and fluid management that is generally accepted common practice - i.e., preoperative fasting guidelines and perioperative fluid administration rates. Subsequent chapters individually cover a particular fluid

V. Tran, MD $(\bowtie)$

Department of Anesthesia, University of Manitoba, Winnipeg, MB, Canada

e-mail: victortran247@gmail.com category, including specific chapters on crystalloid, colloid, glucose-containing, and hypertonic fluids, in addition to blood products. Each chapter starts with theoretical and relevant physiologic considerations before transitioning into an evidence-based approach to clinical applications. I found the chapters regarding colloids and hypertonic solutions to be particularly well written, referencing contemporary and landmark publications that have guided current trends regarding fluids used in clinical practice.

The second section is arguably the most intimidating for the clinically oriented reader as it delves into the basic chemistry and physiology of the fluids used. This aspect is often ignored (or simply forgotten) by most health providers involved in administering fluid therapy. The section opens with a chapter entitled, "Body Volumes and Kinetics" - one of several chapters authored by Hahn himself. It explores the intravascular volume effect of an intravenous fluid bolus as determined by various tracers (i.e., radioactive albumin for plasma volume, iohexol for extracellular fluid space, deuterium for total body water). Hahn also relates these principles to our own endogenous tracer (i.e., hemoglobin) to analyze and simulate the distribution and elimination of infusion fluids. Other sections take a more chemistry-oriented approach to fluids, such as that seen in the chapter, "Acid-Base Issues in Fluid Therapy," which explains the strong ion difference and its significance to crystalloid therapy. Subsequent chapters approach fluids from a more physiologic perspective, such as their interaction with the coagulation system and the microvasculature (including the glycocalyx).

The third section builds on the previous two by transitioning into current practice trends in hemodynamic monitoring of patients. The first few chapters on invasive 
and non-invasive hemodynamic monitoring are wellwritten, introductory chapters targeted at both the senior trainee level and the practicing physician. These chapters build on a well-established understanding of the various types of fluids used in modern medicine and the physiologic principles of how fluids interact in the body. These chapters are supported by up-to-date references and tables that comprehensively summarize the current literature. Furthermore, the tables justify to the reader why the authors support certain practices, such as goaldirected fluid therapy and specific hemodynamic monitoring. Other topics in this section cover hemodilution and enhanced recovery after surgery. Although these chapters could be criticized for being somewhat brief, the ample references at each chapter's end directs those who want further details on the subjects to more-extensive explanations.

The fourth and final section addresses the various clinical environments and situations where fluid management is part of modern practice. It is, by far, the largest section in the book, comprising half of its content. Examples of these chapters' subjects include neuraxial vasoplegia, cardiac surgery, pediatrics, transplantation, neurosurgery, critical care, septic shock, hemorrhagic shock, and burns. The chapters, authored by experts in their respective fields, are written in a manner wherein they can easily be referenced on their own. Thus, the casual reader of this book will be able to refer to these chapters for background knowledge or to review a topic without needing to read the entire book. As with previous chapters, entire textbooks of their own could be devoted to the various subjects included in this section. Hence, they serve as a starting point, or a summary, of the current knowledge on the principles of application.

This second edition of Hanh's textbook serves as a worthy update to the original edition, bringing both new and refreshed content that is relevant to trainees and practicing physicians alike. The book serves as a great teaching tool to expand on topics that may be too detailed to cover adequately during bedside teaching sessions. Thus, it can easily find itself at home on the shelf with other books typically aimed at those who administer fluids, including anesthesiologists, intensivists, surgeons, emergency medicine physicians, and other health care providers who work and train in these areas.

Conflicts of interest None declared.

Editorial responsibility This submission was handled by Dr. Hilary P. Grocott, Editor-in-Chief, Canadian Journal of Anesthesia. 